\title{
Germanica
}

\section{Antimimetischer Realismus}

Programmatik und Praxis der neuen sozialen Dramen

Anti-Mimetic Realism. Principles and Practice of the New Social Drama

Réalisme anti-mimétique. Principes et pratique du nouveau drame social

\section{Georg Feitscher}

\section{OpenEdition}

1 Journals

Édition électronique

URL : http://journals.openedition.org/germanica/2544

DOI : 10.4000/germanica.2544

ISSN : 2107-0784

Éditeur

Université de Lille

\section{Édition imprimée}

Date de publication : 30 juin 2014

Pagination : $39-52$

ISBN : 9782913857339

ISSN : 0984-2632

Référence électronique

Georg Feitscher, « Antimimetischer Realismus », Germanica [Online], 54 | 2014, Online erschienen am: 30 Juni 2017, abgerufen am 06 Oktober 2020. URL : http://journals.openedition.org/germanica/2544 ; DOI : https://doi.org/10.4000/germanica.2544

(c) Tous droits réservés 


\title{
Antimimetischer Realismus Programmatik und Praxis der neuen sozialen Dramen
}

\author{
Georg FEITSCHER \\ Albert-Ludwigs-Universität Freiburg
}

\section{Neues realistisches Engagement}

Wenn im letzten Jahrzehnt eine «Re-Politisierung» 1 des deutschsprachigen Theaters zu beobachten ist, dann ist diese Entwicklung sicher auch als Reaktion auf den drohenden Bedeutungsverlust des Theaters seit den 1990er-Jahren zu verstehen, der in dem katastrophalen Befund gipfelt, «dass das deutsche Drama tatsächlich keine Rolle mehr spielt. Es gibt kein neueres Stück, das in der deutschen Selbstwahrnehmung, in der öffentlichen Kommunikation, eine bedeutsame Stelle markiert hätte» ${ }^{2}$. Der Theaterbetrieb zeigt sich selbstkritisch: Der Autor und

1. - Sabine Sörgel, «Realismus-Variationen. Themen und Tendenzen des Gegenwartstheaters zwischen Glamour, neuer Bürgerlichkeit und Dokumentarismus», in: Friedemann Kreuder / Sabine Sörgel (Hrsg.): Theater seit den 1990er Jahren. Der europäische Autorenboom im kulturpolitischen Kontext, Tübingen 2005, S. 111123, hier S. 119. Allgemein zu den verschiedenen Phasen der Re-Politisierung des deutschsprachigen Theaters nach 1945 vgl. Ingrid Gilcher-Holtey / Dorothea Kraus / Franziska Schößler, «Einleitung», in: dies. (Hrsg.): Politisches Theater nach 1968. Regie, Dramatik und Organisation, Frankfurt a. M. 2006, S. 7-18.

2. - Peter Michalzik, «Dramen für ein Theater ohne Drama. Traditionelle neue Dramatik bei Rinke, von Mayenburg, Schimmelpfennig und Bärfuss», in: Stefan Tigges (Hrsg.), Dramatische Transformationen. Zu gegenwärtigen Schreib- und Aufführungsstrategien im deutschsprachigen Theater, Bielefeld 2008, S. 31-42, hier S. 31 .

GERMANICA, 2014, LIV, pp. 39 à 52 
Dramaturg John von Düffel bemerkt etwa, es gebe «kaum ein Theater, das heute noch gesellschaftlich Position bezieht», und stellt öffentlich die «Selbstbegründung von Theater in Zeiten seiner gesellschaftlichen Entbehrlichkeit» infrage ${ }^{3}$. Und sein Kollege Björn Bicker verlangt, man müsse «das Theater zu einem Ort der produktiven Empörung» gegen politische und soziale Ungerechtigkeit umgestalten, und fragt anschließend: "Welchen angemessenen literarischen Ausdruck kann ich meiner Empörung verleihen?» ${ }^{4}$ Der Fluchtpunkt dieses engagierten Diskurses ist offensichtlich: Es geht um eine neue Rechtfertigung der aufwändigen und kostenintensiven Theaterkunst, die vor allem durch eine stärkere Profilierung des Theaters als kritisches Reflexionsmedium des sozialen, ökonomischen und politischen Status quo erreicht werden soll ${ }^{5}$. Und in dem Maße, wie das Theater um seine gesellschaftskritische Funktion, um sein Publikum und damit letztlich um die überlebensnotwendigen öffentlichen Subventionen fürchtet, gerät auch der Theatertext unter Druck.

Die 〈neuen sozialen Dramen〉 bilden eine der prononcierten Antworten der jungen Autorengeneration auf die gegenwärtige 〈Krise des Theaters〉. Als ausgeprägte Strömung innerhalb des breiten Spektrums der gegenwärtigen Theatertexte zeichnen sie sich durch ihre Orientierung an realistischen Alltagsstoffen und eine Tendenz zu sozial engagierten Botschaften aus. Ihre Entstehung und ihr Erfolg wird von zwei wesentlichen Entwicklungen angetrieben, die bis in die 80er- und 90er-Jahre des 20. Jahrhunderts zurückreichen: Erstens der äußere soziale Strukturwandel hin zu einem «neuen Kapitalismus〉 mit neuen gesellschaftlichen und wirtschaftlichen Krisenphänomenen, die den jungen Theaterautoren die Stoffe liefern ${ }^{6}$. Und zweitens die gewaltigen Verschiebungen innerhalb des Theaterbetriebs im Laufe der letzten zwanzig Jahre. Die Bühnenpraxis in der zweiten Hälfte des 20. Jahrhunderts bewirkte eine Transformation des Theatertextes, der sich von traditionellen dramatischen Elementen wie Handlung, Dialog und Figur zunehmend entfernte und zum Material für performative und <postdramatische> Inszenierungsweisen wurde. ${ }^{7}$ Gegen diese

3. - John von Düffel, «Neue Texte braucht das Land. Programmlosigkeit und Perspektiven - zur Lage der neuen deutschen Dramatik», in: Theater der Zeit, 2000, Jg. 55, H. 10, S. 16-18, hier S. 16.

4. - Björn Bicker, «In Zukunft für das Theater schreiben. Über die Bedeutung von Kontext und Engagement», in: Theater der Zeit, 2011, Jg. 66, H. 7/8, S. 64-69, hier S. 64.

5. - Vgl. Michael Hofmann, «Neue Tendenzen der deutschsprachigen Dramatik», in: Clemens Kammler (Hrsg.): Deutschsprachige Gegenwartsliteratur seit 1989. Zwischenbilanzen - Analysen - Vermittlungsperspektiven, Heidelberg 2004, S. 51-60, hier S. 59.

6. - Vgl. z. B. Richard Sennett, Der flexible Mensch. Die Kultur des neuen Kapitalismus. Aus dem Amerik. von Martin Richter, Berlin 1998.

7. - Vgl. Hans-Thies Lehmann, Postdramatisches Theater, Frankfurt a. M. 1999. 
Entwicklung formulierte 1999 der damalige Jung-Regisseur Thomas Ostermeier einen bis heute wirkungsmächtigen Appell. Seine vielzitierte Forderung nach einem «neuen Realismus», dessen Kern «die Tragödie des gewöhnlichen Lebens» bilden solle, verband Ostermeier mit der Vorstellung von einer (wieder-)erstarkten Rolle von «Autoren», dramatischen «Figuren», «Konflikte[n]» und «Fabeln» ${ }^{8}$. Einige namhafte Autoren und Dramaturgen unterstützten Ostermeiers Position ${ }^{9}$. Andererseits provozierte Ostermeier auch polemischen Widerspruch. Der Dramaturg Matthias Lilienthal verspottete die neorealistische Programmatik mit dem Begriff der «Postpostpostmoderne» und hob dagegen die «Auflösungs- und Fragmentierungstendenzen» sowie die «diskontinuierlichen Wahrnehmungsformen» des postdramatischen Theaters hervor, die einer neuen «Alltagsrealität» entsprächen ${ }^{10}$. Den Kern der Debatte bildete also schnell die Frage, welche Art von Theatertexten heute noch geeignet ist, um soziale Realität angemessen auf der Bühne darzustellen: die auf Ganzheitlichkeit, Illusionismus und Sinnverstehen ausgerichteten Texte des dramatischen Theaters oder doch eher die fragmentarischen, auf sinnliches Erleben sprachlicher Materialität hin konstruierten Texte des postdramatischen Theaters?

\section{Neue soziale Dramen}

Ostermeiers Appell traf den Nerv der Zeit, denn tatsächlich produzieren Theaterautoren seit den späten 1990er Jahren wieder vermehrt Texte, die inhaltlich den Anschluss an gesellschaftliche Krisenphänomene suchen und formal im Zeichen der «Redramatisierung» des Theatertextes stehen ${ }^{11}$ : Traditionelle dramatische Strukturen wie spannende, konflikthafte Handlungen und die Darstellung von Figuren in dialogischer Interaktion erleben eine Renaissance unter jungen Theaterautoren - ohne dass damit jedoch der postdramatische Theatertext vom Tisch gewesen wäre. Sowohl gesellschaftliche Entwicklungen als auch «Konflikte zwischen poetologischen Konzepten» bilden also den Katalysator für das Entstehen einer neuen, realistischen Dramatik ${ }^{12}$. Das Ergebnis dieses Prozesses

8. - Thomas Ostermeier, «Das Theater im Zeitalter seiner Beschleunigung», in: Theater der Zeit, 1999, Jg. 54, H. 4, S. 10-15, hier S. 13.

9. - Vgl. z. B. Düffel, «Neue Texte braucht das Land» (a.a.O.).

10. - Matthias Lilienthal, «Postpostpostmoderne. Vom Cloning des Realismus», in: Theater der Zeit, 2000, Jg. 55, H. 1, S. 14 f., hier S. 14.

11. - Vgl. Stefan Tigges, «Dramatische Transformationen. Zur Einführung», in: ders. (Hrsg.): Dramatische Transformationen. Zu gegenwärtigen Schreib- und Auffiihrungsstrategien im deutschsprachigen Theater, Bielefeld 2008, S. 9-27, hier S. 25.

12. - Vgl. Franziska Schößler, «Avantgarde nach dem Ende der Avantgarde. Soziales Engagement und Aktionskunst nach 1995», in: Ingrid Gilcher-Holtey (Hrsg.): Zwischen den Fronten. Positionskämpfe europäischer Intellektueller im 20. Jahrhundert, 
sind Theatertexte mit konkreterer, stärker an der sozialen Wirklichkeit orientierter, (tages-)aktueller Thematik und einer eingängigen, traditionelleren Rezeptionserwartungen entsprechenden, damit aber auch kommensurableren Ästhetik ${ }^{13}$.

Ihren sichtbarsten Niederschlag fand diese neue Programmatik seit den 1990er Jahren zunächst in den sogenannten 〈Wirtschaftsdramen〉, welche den Fokus auf die wirtschaftlichen Eliten der Gesellschaft legen ${ }^{14}$. Im ersten Jahrzehnt des 21. Jahrhunderts tritt jedoch eine andere Art von Theatertexten hervor, die sich anstelle der ökonomischen 〈Top Dogs〉 wieder den unteren sozioökonomischen Schichten zuwenden. Diese Dramen ähneln formal in vieler Hinsicht den 〈Wirtschaftsdramen schen neuen Realismus zuordnen. Durch ihre inhaltliche Ausrichtung sind sie zudem in einer viel älteren literarischen Filiation zu verorten: Franziska Schößler hat darauf hingewiesen, dass mit den jüngsten Stücken des neuen Realismus nun eine «neue soziale Dramatik» vorliege ${ }^{15}$, die thematisch und oft auch programmatisch den Anschluss an eine dramatische Tradition sucht, die sich von ihren Anfängen bei den bürgerlichen Trauerspielen und einigen Sturm und Drang-Dramen über kanonische Texte wie Büchners Woyzeck und die naturalistischen Pionierdramen bis zu den Volksstücken des 20. Jahrhundert erstreckt ${ }^{16}$. Doch imitierten die jungen Theaterautoren keineswegs bloß etablierte Schemata, sondern gingen innovativ und produktiv mit ihrem Erbe um, etwa indem sie Gattungsvorgaben transformierten, komische und tragische Elemente überlagerten und sich so in der «Hybridisierung von Genres» übten ${ }^{17}$.

\section{Antimimetischer Realismus}

Soziale Problemlagen bildeten in der Theatergeschichte vor allem das Spielfeld einer realistischen oder gar naturalistischen, mimetischen und repräsentationalen Ästhetik. Doch wie lassen sie sich heute mit sozialkritischem Impetus auf eine Theaterbühne bringen, auf der

Berlin 2006, S. 379-396, hier S. 380 f.

13. - Vgl. Joachim Fiebach, Manifeste europäischen Theaters. Grotowski bis Schleef, Berlin 2003, S. 352-354; sowie Sörgel, «Realismus-Variationen» (a.a.O.), S. $121 \mathrm{f}$.

14. - Vgl. Franziska Schößler / Christine Bähr, «Die Entdeckung der «Wirklichkeit〉. Ökonomie, Politik und Soziales im zeitgenössischen Theater», in: dies. (Hrsg.): Ökonomie im Theater der Gegenwart. Ästhetik, Produktion, Institution, Bielefeld 2009, S. 9-20.

15. - Vgl.z. B. Franziska Schößler, Augen-Blicke.Erinnerung, Zeit und Geschichte in Dramen der neunziger Jahre, Tübingen 2004, S. 12 f. sowie dies., «Avantgarde nach dem Ende der Avantgarde» (a.a.O.), S. 381.

16. - Vgl. Theo Elm, Das soziale Drama. Von Lenz, bis Kroetz, Stuttgart 2004.

17. - Schößler, Augen-Blicke (a.a.O.), S. 12. 
nach dem 〈Durchgang〉 durch das Brechtsche und das postdramatische Theater die ungebrochene Verwendung von Techniken der Mimesis und Identifikation naiv und ästhetisch rückständig erscheinen muss? Welche Strategien entwickeln Texte, um realistische und engagiert-appellative Effekte jenseits von Identifikation und Mimesis hervorzurufen?

In einem programmatischen Artikel versuchte Björn Bicker jüngst, einen Ausweg aus diesem Dilemma der neuen sozialen Dramen zu formulieren:

Authentizität besteht im Betonen des Künstlichen und Künstlerischen. [...] Also zu sagen: Das hier ist Kunst. Aber Kunst, die sich die Wirklichkeit aneignet, die mit ihr umgeht, die sich für sie interessiert und sie verändern und sie sich nicht nur als Orden oder Deko ans Revers heften will ${ }^{18}$.

Die zunächst paradox erscheinende Identifikation von Authentizität mit Künstlichkeit, die Bicker vornimmt, wird vor dem Hintergrund eines neuen, konstruktivistischen Begriffs von Realismus verständlich, der die Subjektivität jeglicher Perzeption von Wirklichkeit in Rechnung stellt. ${ }^{19}$ Ein Theatertext müsse mithin die subjektive Perspektive auf die Wirklichkeit, auf der er basiert und die er auf der Bühne reproduziert, von der - tatsächlichen, verborgenen oder angenommenen - Realität unterscheiden und diese Differenz selbstreflexiv thematisieren. Die Künstlichkeit jeglicher Wirklichkeitswahrnehmung und der Prozess ihrer Konstruktion sollen ausgestellt werden.

Dieser Vorschlag Bickers ist anregend, weil er eine kohärente Begründung dafür anbietet, dass die Theatertexte des neuen Realismus - ganz anders als etwa die historischen sozialen Dramen - auf illusionistische Wirkungen nicht nur verzichten, sondern sie gezielt mit künstlichen, antimimetischen Effekten zu unterlaufen versuchen. Denn wie im Folgenden zu zeigen sein wird, realisieren die neuen Theatertexte ihre soziale Appellfunktion nicht mehr, indem sie dem Zuschauer Einfühlung und Identifikation mit den bemitleidenswerten Figuren an der sozialen Peripherie ermöglichen. Stattdessen - so die Ausgangsthese - eröffnen sich den jungen Autoren erst im Spiel mit der Künstlichkeit 〈realistischer〉 Darstellungen Möglichkeiten, die Mechanismen der individuellen oder kollektiven Aneignung von Wirklichkeit explizit vorzuführen. Dazu gehört u. a. auch, dass die neorealistischen Stücke der letzten Jahre sich nicht mehr durch den radikalen Bruch mit postdramatischen Elementen auszeichnen, sondern vielmehr durch deren Integration und Funktionalisierung für die spezifische Wirkungsästhetik

18. - Bicker, «In Zukunft für das Theater schreiben» (a.a.O.), S. 65.

19. - Vgl. dazu die wegweisenden Studien von Wolfgang Preisendanz in ders.: Wege des Realismus. Zur Poetik und Erzählkunst im 19. Jahrhundert, München 1977. 
des Textes. Indem sie Verfahren beider Paradigmen miteinander verschränken, schaffen sie eine neue Ausdrucksform, die hier als <antimimetischer Realismus> bezeichnet wird. Auf diese Weise können die jungen Autoren Wirklichkeitskonstruktionen als problematisch entlarven, neu entwerfen und sogar auf bestimmte Ziele hinlenken - der Realismus verlässt den Bereich des einfach Abbildenden und gelangt in den Bereich der politischen Intervention.

\section{«Ausgegrenzte, Entbehrliche, Überflïssige» 20}

Nis-Momme Stockmanns (*1981) Das blaue, blaue Meer erzählt in einer offen angelegten, relativ unverbundenen Folge von zwölf Szenen die verhinderte Liebesgeschichte zweier junger Menschen. Schon die sprechenden Namen der Figuren verweisen auf ihre randständige Existenz in den Schattenbereichen der Gesellschaft: Der junge Darko verliebt sich in die neunzehnjährige «Wohnsiedlungsprostituierte» namens Motte, die in einem benachbarten Wohnblock im selben Neubaugebiet am Stadtrand wohnt ${ }^{21}$. In anekdotisch-erzählerischen Monologen tritt Darko aus der Handlung heraus, um sich selbst, seine Wohnsiedlung und die dort lebenden Menschen zu charakterisieren - als Vermittler und «Fremdenführer〉 führt Darko das Publikum durch seine Welt. Die Nachbarn in der Siedlung erscheinen in Darkos Darstellung als Opfer eines Prozesses von Verfall und Sinnverlust:

Zu den Menschen hier - Das ist schnell erzählt: Sie wohnen hier, sie leben hier. Sie bewegen ihre kaputten Körper durch die kaputten Straßen. Hier kann man einkaufen, hier wohnen die Familien und Freunde, hier arbeitet man. Man braucht seinen Körper nie irgendwo anders hinzubewegen. Immer hier. Immer hier. Die Siedlung - ein gigantischer Sarg aus Stahlbeton. [...] Als Menschen kommen sie rein, raus kommen sie verrïckt, einsam und zerrissen. So sind sie. Alle 22 .

Die Stadtrandbewohner leben nur innerhalb ihres Wohnviertels und ihrer selbstzweckhaften Alltagsroutinen, ihre Bedeutung für die Gesamtgesellschaft haben sie verloren. «Die Armut, die Enge und Nähe» 23 , unter der sie leiden, stellen die Menschen in der Wohnsiedlung in die Nachfolge eines kanonischen Repräsentanten des massenhaften Pauperismus zu Beginn des 19. Jahrhunderts: Woyzeck (1836/37), mit dem Georg Büchner bittere Armut, beengende Lebensbedingungen und unfreiwillig-intime Nähe als Stoffe für das Drama erschloss und

20. - Vgl. Heinz Bude / Andreas Willisch (Hrsg.): Das Problem der Exklusion. Ausgegrenzte, Entbehrliche, Überflüssige, Hamburg 2006.

21. - Nis-Momme Stockmann, Das blaue, blaue Meer, Köln 2009, S. 10.

22. - Ebd., S. 6.

23. - Ebd., S. 18. 
so erstmals die ökonomischen Bedingungen als unheilvolles Fatum an die Stelle rückte, die bis dahin das «tragische Schicksal〉 eingenommen hatte ${ }^{24}$.

Doch anders als im Falle Woyzecks kämpfen Stockmanns Figuren nicht in erster Linie mit finanzieller und materieller Armut. Zersetzenden Einfluss auf ihre Psyche entfaltet vielmehr das Leben in der Wohnsiedlung selbst. In Das blaue, blaue Meer ebenso wie in vielen weiteren jungen Dramen nehmen die Figuren eine marginale Stellung im sozialen Netz ein und verfügen nicht über die Kompetenzen und Qualifikationen, sich aus ihrer prekären Position zu befreien. Eine Begriffsentlehnung aus der Soziologie bietet sich zur Beschreibung der neuen sozialen Dramen und ihres Personals an: Der Soziologe Heinz Bude prägte vor wenigen Jahren den provokanten Terminus der 〈Überflüssigen〉 für eine neue, heterogene Gruppe, die durch die sozioökonomischen Veränderungen der letzten Jahrzehnte hervortritt: «Gemeinsam ist ihnen der Eindruck des Unnützen und Unverträglichen ihrer Lebenserfahrungen und Lebensbilanzen mit den herrschenden Normierungen und Idealisierungen der Gesamtgesellschaft» ${ }^{25}$.

Die Spezifik der Darstellung dieser 〈Überflüssigen〉 in der jüngsten realistischen Dramatik liegt darin, dass ihr Fokus weniger auf dem Erleiden materieller Not liegt, sondern vielmehr auf einer Kombination von innerem Hoffnungs- und Werteverlust der Figuren und ihrem äußeren Ausschluss von sozialer und ökonomischer Partizipation. Dieser Perspektivwechsel entspricht einer Verschiebung innerhalb der sozialwissenschaftlichen Forschung, die Armut seit mehr als einem Jahrzehnt «stärker im Begriff der Exklusion, des soziokulturellen Ausschlusses von gesellschaftlicher und kultureller Teilhabe, als in Kategorien des materiellen Mangels diskutiert» ${ }^{26}$. Während frühere soziale Dramen diese Ausgestoßenen oft direkt mit ihrer privilegierten Umwelt konstrastierten, bleiben in den neuen Dramen die Exkludierten und 〈Überflüssigen〉 meist unter sich, in einer segregierten 〈Gegengesellschaft〉, die physisch und psychologisch vom restlichen Gesellschaftssystem abgekoppelt zu sein scheint.

24. - Vgl. Alfons Glück, «Der «ökonomische Tod〉. Armut und Arbeit in Georg Büchners Woyzeck», in: Thomas Michael Mayer / Hubert Gersch / Günter Oesterle (Hrsg.): Georg Büchner Jahrbuch, Bd. 4, Berlin/New York 1984, S. 167-226, hier v. a. S. 194.

25. - Heinz Bude / Andreas Willisch, «Die Debatte über die 〈Überflüssigen〉. Einleitung», in: dies. (Hrsg.): Exklusion. Die Debatte über die »Überflüssigen «, Frankfurt a. M. 2008, S. 9-30, hier S. 30.

26. - Elke Brüns, «Einleitung. Plädoyer für einen social turn in der Literaturwissenschaft», in: dies. (Hrsg.): Ökonomien der Armut. Soziale Verhältnisse in der Literatur, München 2008, S. 7-19, hier S. 11. Vgl. außerdem Heinz Bude / Andreas Willisch, «Das Problem der Exklusion», in: dies. (Hrsg.): Das Problem der Exklusion. Ausgegrenzte, Entbehrliche, Überflüssige, Hamburg 2006, S. 7-23. 
Bei der Beschreibung der Stadtrandbewohner in Das blaue, blaue Meer entfaltet Darko ein Tableau überzeichneter Stereotypen: «Kahlrasierte Schädel schieben morgens Kinderwagen durch die Siedlung. Tarnklamotten, Stahlkappen und in der rechten Hand ein Bier» ${ }^{27}$, berichtet Darko über die Neo-Nazis in der Siedlung, die aber im Vergleich mit den «Satanisten» noch harmlos erscheinen: «Die haben hier letztens einen 8-jährigen Jungen vom Spielplatz gelockt. Aufgemacht. Ein bisschen was von dem gegessen. Voll wenig! Dann ist ihnen schlecht geworden» 28 .

Unermüdlich protokolliert und referiert Darko die Gewaltexzesse in der Siedlung. Im Gegensatz zu dem auf Schockwirkung abzielenden britischen 〈Brutalism〉 der 1990er Jahre funktionalisiert Stockmann in seinem Stück die Gewaltdarstellung jedoch auf zwei andere Weisen. Erstens bricht er die grotesk übertriebene Schilderung der Gewalthandlungen durch ironische Pointen, die oft auf eine makabre Komik abzielen. So erklärt Darko: «Manfred hat 20 Jahre lang Beine von Schaufensterpuppen geklaut, auf die er masturbiert hat. Das hat ihm aber irgendwann nicht mehr gereicht. Ich lass das so stehen» 29 .

Zweitens gewinnen Darkos Anekdoten der Gewalt manchmal poetische Qualitäten ab, die zu der Brutalität der Handlungen in einem merkwürdigen Kontrast stehen:

Darko: [...] Hier, genau wo wir gerade stehen [...] ist Frau Jenowitsch verbrannt. Ihr Mann hat sie, als sie schlief, angezündet. [...] Man konnte sie über den ganzen Kilometer der Siedlung schreien hören. Und es gab eine gigantische kegelförmige Rauchsäule. Man konnte sie in der Stadt sehen und sogar in den Kellern riechen. Irgendwie war ich neidisch. $\mathrm{Zu}$ Teilen lag sie da unten, kaputt, zu anderen Teilen beendete sie aber gerade ihr trauriges Dasein, indem sie über den Dächern tanzte ${ }^{30}$.

Darko verklärt das morbide Bild eines brennenden Menschen zu einer poetischen, in ihrem Symbolismus geradezu kitschigen Imagination. Dieses unvermittelte Nebeneinander von Brutalität, grotesker Komik und poetischer Transzendenz prägt Stockmanns Text beinahe durchgängig.

Stockmanns Das blaue, blaue Meer ist ein drastisches Beispiel für die Versuche jüngerer Autoren, die tragischen Geschichten der Exkludierten und 〈Überflüssigen〉 weiterhin für ihre dramatischen Texte zu nutzen, sich jedoch neue Darstellungsweisen für diese Stoffe zu erschließen. In Zeiten, in denen «das Arbeitslosendrama [...] nicht mehr

27. - Stockmann: Das blaue, blaue Meer (a.a.O.), S. 26.

28. - Ebd., S. 17.

29. - Ebd., S. 25.

30. - Ebd., S. 23. 
als Tragödie erzählt werden kann» und realistische Darstellungsweisen suspekt geworden sind ${ }^{31}$, setzen die Autoren nicht länger auf traditionelle Wirkungsabsichten wie Schock und Mitleid, sondern inszenieren soziale Problemlagen im spannungsvollen Verhältnis von derb-komödiantischem Verlachen und sentimentalischer Verklärung.

\section{Zwischen naturalistischer Mimesis und postdramatischer Autonomie}

Ähnlich kontrastreiche Hybridisierungen heterogener Darstellungsmittel lassen sich in vielen neuen Stücken auf der Ebene der Sprachverwendung wiederfinden. Ein virtuoses Spiel mit Registerund Stilbrüchen entfaltet insbesondere Dea Loher (*1964). Ihr Theatertext Das letzte Feuer schildert in 33 lose montierten Szenen das Schicksal von acht unter Arbeitslosigkeit und Krankheit leidenden Personen in einer heruntergekommenen Wohnsiedlung, die sie selbst als «Glasscherbenviertel ohne Zukunft» bezeichnen ${ }^{32}$. Im Zentrum der Handlung steht ein tragischer Verkehrsunfall, in dem ein Knabe sein Leben verliert und in den die übrigen Figuren auf verschiedene Weise involviert waren. Häufig tendiert ihre Rede zur naturalistischen Mimesis mündlicher Umgangssprache, die das Milieu der gezeigten Personen abbildet oder gar parodistisch überhöht:

[...] ich hab das Kind überfahren, weil dein Kerl $\mathrm{n}$ durchgeknallter Koksarsch is, der mit hundert durch die Stadt rauscht und nich weiß, wo die Bremse is und am Ende so kaputt is, dass er die Kiste gegen ne Scheißhauswand setzt $[\ldots]^{33}$.

Doch wechselt die Figurenrede häufig abrupt in einen artifiziellen, denaturalisierenden Sprachmodus und bedient sich lyrisch-verdichteter Bilder. Die Materialität der Sprache tritt hervor und überlagert die semantische Dimension des Gesagten. In Lohers Das letzte Feuer sind diese Passagen meist nicht Teil der Figurenrede, sondern durchbrechen immer wieder als kollektiver Erzählerbericht eines das gesamte Personal einschließenden «WIR» die Figurenrede:

Wir, die wir diese Geschichte erzählen

Uns gibt es womöglich gar nicht

Wir, als die Gemeinschaft, die wir vorgeben zu sein

Uns gibt es gar nicht

31. - So John von Düffel in Franziska Schößler, «Interview mit John von Düffel. (25. März 2003 am Thalia Theater Hamburg)», in: dies.: Augen-Blicke. Erinnerung, Zeit und Geschichte in Dramen der neunziger Jahre, Tübingen 2004, S. 315-324, hier S. 319.

32. - Dea Loher, Das letzte Feuer, Frankfurt a. M. 2008, S. 42.

33. - Ebd., S. 27. 


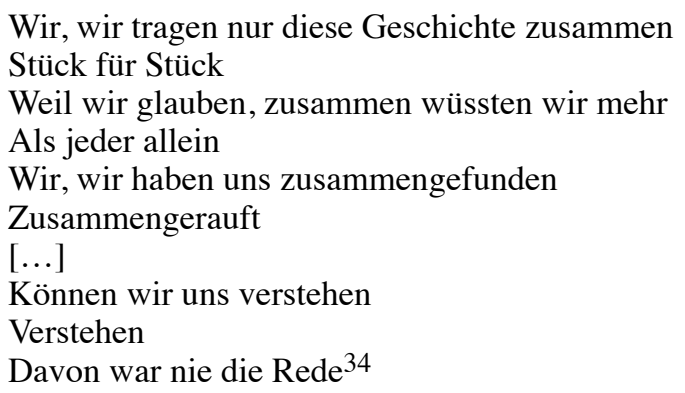

Dem Zuschauer tritt eine Art 〈lyrisches Wir〉 gegenüber, dessen Äußerungen nicht die Dramenhandlung vorantreiben, sondern die Bedingung seines eigenen Seins reflektieren. Die starke Betonung selbstreferentieller Sprachstrukturen gründet in der Rhythmizität des Wechsels von langen Hauptsätzen und kurzen parenthetischen Nachschüben, in der Lautlichkeit des anaphorischen «Wir, [...]» und nicht zuletzt in der leitmotivischen Betonung des Kollektivs (〈wir〉, 〈uns〉 und (zusammen〉). So entsteht schließlich ein verdichtetes Textgebilde, das sich mit Stricker als ein «Theatertext» beschreiben lässt, «der nicht mehr nur Sprechtext und szenische Anweisungen vorsieht, sondern den Charakter einer bildnerischen Sprachbehandlung oder musikalischen Partitur annehmen kann»35. In der Aufführung des Textes kommt es zu der für das postdramatische Theater typischen «Autonomisierung der Sprache und des Sprechens», d. h. einer Verschiebung der Aufmerksamkeit der Zuschauer von der Semantik des Textes hin zur Wahrnehmung der akustischen Qualitäten des auf der Bühne Gesprochenen ${ }^{36}$.

Darüber hinaus bricht Loher mit weiteren traditionellen Merkmalen des Dramatischen, und zwar vor allem mit der Konstitution des dramatischen Textes aus Figurenrede und Nebentext. In den Textabschnitten des 〈WIR〉 lassen sich verschiedene Stimmen ausmachen, die sich gegenseitig ergänzen, verbessern, widersprechen. Doch weist Loher diesen Stimmen keine Figuren oder Rollen zu und gibt nicht einmal typografische Hinweise auf Sprecherwechsel. Diese Trennung von Text und Sprecher hält Loher in einer Anmerkung unter dem Personenverzeichnis explizit fest: «Der Text von WIR soll nie chorisch gesprochen werden, sondern aufgeteilt in Einzelstimmen. Der Sprecher bzw. die Figur wird

34. - Ebd., S. 16.

35. - Achim Stricker, Text-Raum. Strategien nicht-dramatischer Theatertexte. Gertrude Stein, Heiner Müller, Werner Schwab, Rainald Goetz, Heidelberg 2007, S. 35. (Hervorhebung i. O.)

36. - Lehmann, Postdramatisches Theater (a.a.O.), S. 266. (Hervorhebung i. O.) 
nicht immer benannt; wo die Zuordnung nicht aus dem Zusammenhang hervorgeht, ist sie der Regie überlassen» ${ }^{37}$.

Loher löst so zwar die dialogische Oberflächenstruktur des Textes auf, untersagt jedoch die - im postdramatischen Theater häufig praktizierte - chorische Wiedergabe des Textes. Stattdessen nimmt sie den Regisseur in die Verantwortung, im Laufe des Inszenierungsprozesses den Textblock wieder in Einzelstimmen zu zerlegen und bestimmten Figuren zuzuweisen. Damit folgt Loher einerseits einem postdramatischen Verständnis des Textes als bloßem «Material», das im Laufe der Inszenierung modifiziert und nutzbar gemacht wird. Andererseits lässt sich ihr Insistieren auf einzelnen Sprecherstimmen als beinahe programmatischer Gegenentwurf zur postdramatischen Ästhetik begreifen. Bezeichnet Lehmann es als «symptomatisch für das postdramatische Theater [...], daß die dialogische Struktur zugunsten von monologischen und chorischen abgelöst wird» ${ }^{38}$, so schlägt Loher in Das letzte Feuer den entgegengesetzten Weg ein: Aus dem monolithischen Textblock treten die einzelnen Stimmen wieder hervor, aus dem monologischen oder chorischen 〈WIR〉 im Text entsteht auf der Bühne wieder ein Dialog einzelner Figuren.

Lohers Beispiel zeigt, wie die neuen sozialen Dramen sowohl naturalistische als auch postdramatische Verfahren für ihre Zwecke funktionalisieren: Naturalistische Dialoge finden sich häufig in Situationen, in denen die Gegenwärtigkeit des Geschehens und die Perspektive der an ihr beteiligten Personen in den Vordergrund rücken. Oft wird diese Perspektive jedoch durch kommentierende und abstrahierende Metadiskurse durchbrochen, die auf dialogische Handlungsstrukturen weitestgehend verzichten und durch lyrische Verdichtung, den intensiven Einsatz rhetorischer Tropen oder der Betonung klanglicher Qualitäten eine künstliche und antimimetische Sprache zur Geltung bringen. Durch das komplexe Spiel mit sprachlichen Ausdrucksmitteln propagieren die Theatertexte einerseits ihr realistisches Interesse an gesellschaftlicher Wirklichkeit, problematisieren aber andererseits selbstreflexiv die Möglichkeiten des adäquaten Beschreibens und Erzählens dieser Wirklichkeit.

\section{Pseudosoziologische Diskurse}

Lohers ambivalenter Umgang mit postdramatischen Theaterpraktiken ist kein Einzelfall. So finden sich auch in Ewald Palmetshofers (*1978) tier. man wird doch bitte unterschicht Momente der Auflösung dialo-

37. - Loher, Das letzte Feuer (a.a.O.), S. 8.

38. - Lehmann, Postdramatisches Theater (a.a.O.), S. 233. 
gischer Figurenrede und fester Sprecheridentitäten ${ }^{39}$. Die Renaissance des antiken Chors im postdramatischen Theater greift Palmetshofer auf parodistische Weise auf, indem er den Figuren verschiedene Stimmfächer eines musikalischen Chores zuordnet. Die Stimmen nehmen im Laufe des Textes jeweils Rollen in drei distinkten Geschehensebenen an, die Palmetshofer durch verschiedene Schrifttypen markiert. Zusätzliche Komplexität gewinnt diese Konstruktion dadurch, dass die Figuren in allen drei Ebenen von einer einzigen Gruppe von Schauspielern gespielt werden sollen, wie das Personenverzeichnis verdeutlicht ${ }^{40}$ :

$\begin{array}{ll}\text { CHOR: } \\ \text { SOPRAN } & \begin{array}{l}\text { Expertin / Greißlerin / Kind } \\ \text { Expertin / Freundin / Kind }\end{array} \\ \text { ALT } & \begin{array}{l}\text { Experte / Stammgast / Kind } \\ \text { COUNTER }\end{array} \\ \text { TENOR } & \begin{array}{l}\text { Experte / Direktorssohn / Kind } \\ \text { BARITON }\end{array} \\ \text { Experte / Freundinnengatte / Kind } \\ \text { BASS } & \text { Experte / alter Direktor / Direktor }\end{array}$

Palmetshofers Stück setzt auf eine drastische, brutal zugespitzte Handlung: Hauptfigur Erika, die in ihrer Schulzeit von den Mitschülern vergewaltigt wurde, rächt sich am Direktor der Schule (der wiederum einst die Schüler sexuell missbrauchte), indem sie ihn bei lebendigem Leib in seinem Haus verbrennt. Doch neben den Handlungssträngen in Gegenwart und Vergangenheit erweitert Palmetshofer - wie schon Dea Loher mit dem kollektiven 〈WIR〉 und Nis-Momme Stockmann mit den Monologen Darkos - die dramatische Handlung um eine, dritte, reflexive Ebene: Eine Gruppe von «Experten» reist aus dem gesellschaftlichen «Kern» in einen «Ort am Rand, irgendwo» 41 , um dort im Gestus des Chors der attischen Tragödie - das Geschehen auf der Handlungsebene $\mathrm{zu}$ beobachten und die sich offenbarenden sozialen Zerfallsprozesse zu kommentieren.

Beim ersten Auftritt in der Rolle der «Experten〉 schildert der Chor ausführlich seine Motivation, sich mit dem 〈Rand〉 der Gesellschaft auseinanderzusetzen. In der für Palmetshofers Texte typischen, von Ellipsen, Wiederholungen, Anakoluthen und übertriebener Metaphorik geprägten Sprache richten sich die >Experten< direkt ad spectatores ${ }^{42}$ :

39. - Ewald Palmetshofer, tier. man wird doch bitte unterschicht. Frankfurt a. M. 2010.

40. - Ebd., S. 3.

41. - So die vom Autor verfasste Stückbeschreibung, nachzulesen u. a. auf den Seiten des Goethe-Instituts. Vgl. Ewald Palmetshofer, [Stückbeschreibung zu] tier. man wird doch bitte unterschicht, 2010, URL: http://www.goethe.de/kue/the/nds/nds/aut/ $\mathrm{plm} / \mathrm{stu} / \mathrm{de} 8580240 . \mathrm{htm}$.

42. - Palmetshofer, tier (a.a.O.), S. 10-12. 


\begin{tabular}{|c|c|}
\hline ALT & ja, wir fragten immer nach dem Kern \\
\hline BASS & $\begin{array}{l}\text { man möcht' fast sagen Kernphysik im Sozialen, die } \\
\text { wir da betreiben }\end{array}$ \\
\hline BARITON & rein beobachtend natürlich \\
\hline COUNTER & bis das Interesse \\
\hline SOPRAN & uns'res \\
\hline BASS & $\begin{array}{l}\text { an dem Kern } \\
{[\ldots]}\end{array}$ \\
\hline COUNTER & $\begin{array}{l}\text { bis es sich von dieser Kernphysik im Sozialen } \\
\text { abgewandt }\end{array}$ \\
\hline ALT & $\begin{array}{l}\text { das Interesse } \\
{[\ldots]}\end{array}$ \\
\hline TENOR & $\begin{array}{l}\text { dass wir uns dann der Schale, nicht dem Kern } \\
\text { der Fäulnis in den Randbezirken }\end{array}$ \\
\hline $\mathrm{N}$ & in der Hülle um den Kern herum \\
\hline TENOR & dass wir uns schließlich der dann zugewandt \\
\hline
\end{tabular}

Das eigentliche Interesse der 〈Experten〉 gilt dem «Kern», der Mitte der Gesellschaft, mit der sie sich identifizieren. Doch wird der Blick vom Zentrum abgelenkt: Das Bewusstsein für die potenzielle Bedrohlichkeit der sozialen Zerfallsprozesse in der «Schale», an den Rändern der Gesellschaft, nötigt die 〈Experten〉 zur Beobachtung der sozialen Peripherie, weil nämlich «an der Schale schon Verwesung / ekelhaft / ganz braun und faul / ein Loch sich meuchlings in die Mitte frisst» ${ }^{43}$. Explizit formulieren sie ihre Angst vor dem gesellschaftlichen Kollaps: «und würd' der Rand dann in die Mitte stürzen [...] / würd der Kern dann implodier'n [...] / das ist dann Kernfusion, würd' ich mal sagen» ${ }^{44}$.

Bezeichnenderweise benutzen die ¿Experten〉 kein originär soziologisches Vokabular, sondern strapazieren eine aus Physik und Biologie entlehnte Metaphorik, die durch die Suche nach immer neuen Bildern beinahe zur Katachrese verkommt ${ }^{45}$. Palmetshofer erhebt so den szientistischen Diskurs zum integralen Bestandteil seines Textes, doch strebt der unangemessene und häufig ironisch pointierte Einsatz der naturwissenschaftlichen Metaphern selbstverständlich keine affirmative Bezugnahme auf eine vorgeblich wissenschaftliche Beschreibungssprache an. Er verhindert vielmehr die Identifikation mit den Äußerungen der 〈Experten〉. Besonders deutlich wird dies

43. - Ebd., S. 13.

44. - Ebd., S. 70.

45. - Im Rollentext der 〈Experten〉 finden sich neben den schon zitierten Begriffen der «Kernphysik», «Kernfusion», «Schale», «Fäulnis» und «Verwesung» z. B. noch Begriffe wie «Fruchtfleisch», «Same» (S. 9 f.), «Blüten», «Pflanze» (S. 15), «Kettenreaktion», «Wirt», «Infektion» (S. 18 f.), «Reifung» (S. 48), «Attraktion», «Fliehkraft», «Magnetismus» (S. 69). 
an einigen Stellen, an denen sich faschistische Metaphern in die Rede der 〈Experten〉 einschleichen, z. B. wenn sie beklagen, dass es «der Volkshygiene - schlimmes Wort / [...] nicht gelungen ist, das Trägerelement zu isolier'n und auszumerzen» ${ }^{46}$.

Auf diese Weise erzeugt Palmetshofer eine anhaltende Irritation auf Seiten des Publikums: Beherrschen die Figuren die Sprache oder ist es andersherum? Ist die Sprache nur geborgt, zitiert fremde Diskurse, um Wahrnehmung in Worte zu kleiden? Die formalen und inhaltlichen Auflösungserscheinungen, die die Figurenrede in Palmetshofers Stücken prägen, sind jedenfalls ein kaum zu übersehender Hinweis, dass die Figuren nicht über geeignete Ausdrucksmittel verfügen, um ihre soziale und private Situation adäquat zu verbalisieren. Die Sprache verstellt eher den Zugang zur sozialen Wirklichkeit, als dass sie ihn eröffnen würde. Schon der elliptische Untertitel des Textes, «man wird doch bitte unterschicht», der die Ergänzung «... sagen dürfen» provoziert, aber eben nicht explizit formuliert, erhebt diese Aporie zum zentralen Thema des Dramas. So stellt Palmetshofers Stück aus, dass die Perzeption und Repräsentation sozialer Realität immer den Bedingungen der Sprache unterworfen und oft von fremden Diskursen kontaminiert sind - die Sprachskepsis weitet sich zu einer Kritik der gegenseitigen Wahrnehmung von gesellschaftlich Inkludierten und Exkludierten.

Die «neuen sozialen Dramen〉 reagieren auf die jüngste Legitimationskrise des Theaters, indem sie nach empirischer Sättigung streben und diesen Empirismus zugleich selbstreflexiv problematisieren. Ihre Hinwendung zu sozialen Problemlagen erkaufen sich die Autoren also nicht durch ästhetische und programmatische Rückschritte. Statt in naiv-realistische Darstellungsmodi zurückzufallen, praktizieren sie einen <antimimetischen Realismus〉, der eine mimetische und identifikatorische Darstellung der Wirklichkeit gezielt unterläuft. Realismus meint bei den jungen Autoren vor allem die Problematisierung des Blickens auf und des Redens über soziale Exklusionsprozesse. Ihr politisches und sozialkritisches Engagement zielt nicht auf Mitleid mit den 〈Überflüssigen〉 oder Exkludierten, sondern vielmehr darauf, Wahrnehmungsmechanismen zu entlarven, die nicht einfach Effekt sozialer Segregation sind, sondern selbst konstitutiv dafür sind. 